\title{
Osteopathic Treatment of Infants in Their First Year of Life: A Prospective Multicenter Observational Study (OSTINF Study)
}

\author{
Florian Schwerla $^{a}$ Boris Daake ${ }^{b}$ Eva Moeckel ${ }^{c}$ Karl-Ludwig Resch ${ }^{d}$ \\ ${ }^{a}$ German Academy of Osteopathy (AFO), Gauting, Germany; ${ }^{b}$ Practice for Osteopathy, Bonn, Germany; ${ }^{\text {cPractice for }}$ \\ Osteopathy, Hamburg, Germany; ${ }^{\mathrm{d}}$ German Institute for Health Research (DIG), Bad Elster, Germany
}

\section{Keywords}

Osteopathic medicine · Infants · Asymmetry · Plagiocephaly · Feeding and sleeping disorders - Excessively crying infants

\begin{abstract}
Introduction: In Germany in recent years, a growing number of parents are seeking help from osteopaths for the perceived health complaints of their infants and children. However, reliable evidence for the effectiveness of osteopathic interventions for this group of patients is largely lacking. $\mathbf{O b}$ jective: To observe and document changes in the symptoms of certain health disturbances, as perceived by parents, during the course of an osteopathic treatment of their baby, and associated side effects. Methods: A single-arm, prospective, multicenter, observational study was chosen as the study design. Parents who contacted an osteopathic practice with any of the following 5 health disturbances were invited to include their baby into the study: idiopathic infant asymmetry (IA), deformational plagiocephaly (DP), feeding disorders (FD), excessive crying (EC), and sleep disorders (SD). Osteopaths performed osteopathic treatment as usual for these conditions, and the parents were asked to rate the perceived severity of symptoms on standardized forms including numerical rating scales (NRS 0-10). In DP, the head circumference of the child's skull was measured with a craniometer, and the cranial vault index (CVAI) was calculated. Results: 230 osteopaths agreed to participate, 151 osteopaths returned parental ratings. 1,136 infants were treated $2.8 \pm 1.1$ times (mean $\pm \mathrm{SD}$ ). IA was the most prevalent disturbance (48\%), followed by EC (18\%), FD (15\%), DP (14\%), and SD
\end{abstract}

(4\%). In IA, perceived asymmetry as rated on the NRS improved from 6.1 to 1.1 , a mean improvement of $82 \%$. In DP, the CVAl improved from 8.0 to $4.0 \%$, a mean improvement of $51 \%$. FD improved by $77 \%$, EC by $70 \%$ (from 7.7 to 2.3 on the NRS) and SD by $56 \%$. Adverse reactions with a timely association with osteopathic treatments were reported in 3.5\% of all treatments, probably reflecting typical day-to-day variations in symptoms. In a total of 3,212 treatments, there was not a single serious adverse reaction affecting infant health. Conclusions: This is one of the largest studies worldwide to date on the osteopathic treatment of infants. Osteopathic treatment was associated with major positive changes in the severity of perceived health complaints as assessed by parents, which in most cases were resolved as a matter of concern, and was proved to be a safe treatment modality among a large number of therapists. Based on these results, confirmatory intervention studies can and should be planned and conducted.

๑) 2021 S. Karger AG, Basel

Die osteopathische Behandlung von Säuglingen im ersten Lebensjahr: Eine prospektive multizentrische Beobachtungsstudie (OSTINF-Studie)

\section{Schlüsselwörter}

Osteopathische Medizin · Säuglinge · Asymmetrie . Plagiozephalie · Fütter- und Schlafstörungen . Schreikinder 


\section{Zusammenfassung}

Einleitung: In Deutschland suchen in den letzten Jahren immer mehr Eltern bei Osteopathen Hilfe für die wahrgenommenen gesundheitlichen Beschwerden ihrer Säuglinge und Kinder. Verlässliche Belege für die Wirksamkeit osteopathischer Interventionen für diese Patientengruppe fehlen jedoch weitgehend. Studienziel: Beobachtung und Dokumentation von Veränderungen der Symptome bestimmter, von den Eltern wahrgenommener Gesundheitsstörungen im Verlauf einer osteopathischen Behandlung ihres Babys und der damit verbundenen Nebenwirkungen. Methoden: Als Studiendesign wurde eine einarmige, prospektive, multizentrische Beobachtungsstudie gewählt. Eltern, die sich mit einer der folgenden 5 Gesundheitsstörungen an eine osteopathische Praxis wandten, wurden eingeladen, ihr Baby in die Studie einzubeziehen: idiopathische Säuglingsasymmetrie (IA), DeformationsPlagiozephalie (DP), Fütterungsstörungen (FD), exzessives Schreien (EC) und Schlafstörungen (SD). Osteopathen führten die osteopathische Behandlung wie üblich bei diesen Erkrankungen durch, und die Eltern wurden gebeten, den wahrgenommenen Schweregrad der Symptome auf standardisierten Formularen einschließlich numerischer Bewertungsskalen (NRS 0-10) zu bewerten. Bei der DP wurde der Kopfumfang des Schädels des Kindes mit einem Kraniometer gemessen und der Cranial Vault Index (CVAI) berechnet. Ergebnisse: 230 Osteopathen erklärten sich zur Teilnahme bereit, 151 Osteopathen gaben elterliche Bewertungen zurück. 1'136 Säuglinge wurden 2,8 $\pm 1,1$ Mal behandelt (Mittelwert \pm SD). IA war die am häufigsten auftretende Störung (48\%), gefolgt von EC (18\%), FD (15\%), DP (14\%) und SD (4\%). Bei der IA verbesserte sich die wahrgenommene Asymmetrie, wie sie auf der NRS bewertet wurde, von 6,1 auf 1,1, was einer durchschnittlichen Verbesserung von $82 \%$ entspricht. Bei der DP verbesserte sich der CVAl von 8,0 auf 4,0\%, was einer durchschnittlichen Verbesserung von $51 \%$ entspricht. Die FD verbesserte sich um 77\%, die EC um 70\% (von 7,7 auf 2,3 auf der NRS) und die SD um 56\%. Unerwünschte Reaktionen, die in Verbindung zu gleichzeitigen osteopathischen Behandlungen standen, wurden bei 3,5\% aller Behandlungen berichtet, spiegeln aber typische alltägliche Symptome wider. Bei insgesamt 3,212 Behandlungen gab es keine einzige schwerwiegende Nebenwirkung, die die Gesundheit des Säuglings beeinträchtigte. Schlussfolgerungen: Dies ist eine der bisher weltweit größten Studien über die osteopathische Behandlung von Säuglingen. Die osteopathische Behandlung ging mit großen positiven Veränderungen in der Schwere der von den Eltern wahrgenommenen Gesundheitsbeschwerden einher und erwies sich als sichere Behandlungsmodalität. Auf der Grundlage dieser Ergebnisse können und sollten konfirmatorische Interventionsstudien geplant und durchgeführt werden.

\section{Introduction}

The 2010 WHO benchmarks for training in osteopathy point out that "growing numbers of patients are seeking access to osteopathic health care, and more countries are now recognizing osteopathic manipulative medicine (OMM) within their regulatory and national health systems" [1]. In this context, osteopaths have also increasingly been working in the field of pediatrics. According to a survey in 2018 [2], approximately 1.2 million infants in the 0 - to 2 -year age group in Germany have already been treated by an osteopath. The two largest German osteopathic professional associations require additional postgraduate training for osteopaths working with children (e.g., preconditions to be listed as "specialized in treating infants," https://www.osteopathie.de/vodnet). Increasingly, midwives send newborns to an osteopath for checkups (baby tips: 11 best hints of experienced midwifes, https://www.familie.de/baby/hebammen-tipps-fuer-diebabyzeit/).

The growing popularity of osteopathy in Germany has also caused increased attention in the media. For example, an article in the scientific section of the renowned German weekly magazine Die Zeit (No. 33, August 4, 2016) on the subject of osteopathic treatment for children triggered a controversial discussion. The basic tenor of the article was: osteopathy is a dangerous treatment for newborns and infants.

Likewise, the authors of a systematic analysis in the journal Pediatrics in 2013 stated: "The effectiveness of osteopathic manipulation treatment (OMT) for pediatric conditions remains unproven. The low methodological quality and paucity of the primary studies are remarkable. More robust RCTs are needed to clarify the many open questions regarding the effectiveness of OMT. Until such data are available, OMT cannot be considered an effective therapy for pediatric conditions, and osteopaths should not claim otherwise" [3].

Indeed, few clinical studies have been published worldwide on the effectiveness of osteopathic treatment on newborns and infants in the first year of life investigating the topics of excessive crying/infantile colic $[4,5]$ and infantile postural asymmetry/congenital, muscular torticollis [6-10].

Against this background, we found that there are no reliable figures on the typical spectrum of infant disorders for which parents seek advice and help from osteopaths. We were only able to identify two smaller studies exploring this topic $[11,12]$.

The German Academy of Osteopathy (AFO), whose aim it is to promote, create, and publish scientific evidence-based research on osteopathy, therefore placed the topic of osteopathic treatment of infants at the top of its research agenda in 2016. Essentially, the term "infants" 
lacks an authoritative definition as to the exact age range. The AFO has been considering infants in this context as newborns and infants up to 1 year of age, and this definition has been used for the "OSTINF study" (acronym for Osteopathic Treatment of Infants) and for this communication.

In a first step, the spectrum of typical infant health disturbances, for which parents present their child to an osteopath, was investigated [13]. In this survey, no quantitatively dominant health disturbance emerged. Instead, 5 different health disturbances occurred similarly frequently: idiopathic infant asymmetry (IA; 21\%), deformational plagiocephaly (DP; 17\%), excessive crying (EC; 16\%), feeding disorders (FD; 14\%), and sleeping disorders (SD; $14 \%)$.

In preparation for this survey, we realized that these "diagnoses," clinically taken for granted, are in fact not based on a clear pathophysiological concept [e.g., 14, 15]. Hence, through extensive literature analyses, we attempted to develop manageable, best-evidence-based definitions to be made available to all osteopaths involved in this study. For the present paper, the following essential aspects of each health disturbance are therefore briefly presented:

In the literature, there is no consensus on definition, nomenclature, and classification of "infant asymmetry" [14]. The asymmetry is either idiopathic (IA) or symptomatic with a probable prevalence of 1 in 10 [16]. In most cases, the origin seems to be idiopathic.

Plagiocephaly can be divided into two groups: synostotic plagiocephaly, with one or more fused cranial sutures, and nonsynostotic (deformational) plagiocephaly [17]. DP is characterized by changes in skull shape that result from mechanical forces prenatally or postnatally [18]. Since the American Academy of Pediatrics Task Force on Infant Positioning and Sudden Infant Death Syndrome recommended in 1992 that healthy infants be positioned supine for sleeping, the incidence of sudden infant death syndrome has decreased. As a consequence, positional skull deformity became ever more dominant in the literature [19] with 1 in 5 infants being affected to some degree [20]. To date DP is a common problem seen by pediatricians and physical therapists [21]. While DP was formerly considered a cosmetic problem, recent research suggests that infants with the condition may also experience relevant developmental difficulties [22, 23].

Excessive infant crying, in the literature also described as EC and/or infantile colic (in Germany often called "three-month colic"), is a common problem during the first few months of childhood. Studies have reported incidence rates that vary widely from 2 to $40 \%$ [15]. In research studies, the condition is typically defined as crying that lasts for at least $3 \mathrm{~h}$ a day and occurs at least 3 days per week over a period of at least 3 weeks [24]. The etiol- ogy of EC remains unclear, and although it has been appreciated as a manifestation of normal infant behavior and development, an effective treatment is lacking [25, 26].

Feeding is one of the most important interactions between caregiver and child. In the management and treatment of FD, it is extremely important to differentiate between organic and nonorganic causes of the feeding problem. Nonorganic FD are a condition without underlying organic disease [27].

Nonorganic sleep-onset and night-waking disorders are among the most frequent problems of behavioral regulation in the first few years of life. Pediatricians are confronted with a spectrum of various degrees of severity [28, 29]. The German Society for Child and Adolescent Psychiatrists defines sleep disturbances as more than 3 nocturnal wakes in at least 4 nights of the week, combined with the inability to fall asleep again without parental help [30].

\section{Objective}

The results of a survey conducted as part of this study and about to be published elsewhere [13] served as an important basis for the conceptual development of the present study, which should generate quantitatively reliable answers to the following core question:

What changes in the symptoms associated with these 5 most common infant health disturbances in the first year of life are being observed by parents or, with DP, being measured by therapists throughout osteopathic sessions?

In addition, this study aimed at mapping and documenting key features of the course of treatment and the incidence of adverse reactions.

\section{Methods}

\section{Study Design}

The methodology of a single-arm, prospective, multicenter, observational study with pre- and postintervention measures was chosen. The quantitative goal was to document the treatment of at least 500 infants in a wide spectrum of osteopathic practices.

\section{Eligibility Criteria}

Inclusion criteria were:

- Neuropediatrically inconspicuous babies, born after the 37th week of pregnancy, both sexes, aged 0-12 months

- Parents who approached an osteopathic practice with any of the following 5 health disturbances of their child: IA, DP, EC, $\mathrm{FD}$, and $\mathrm{SD}$

- Informed consent and sufficient knowledge of the German language by at least one parent

- Participating therapists: "Heilpraktiker"/physicians with osteopathic training according to WHO standards and defined postgraduate training in the treatment of infants 
Fig. 1. Cranial asymmetry screening mea-

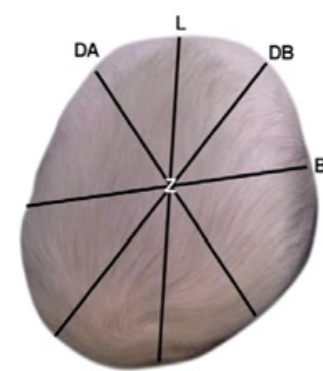

Skull length (L), skull width (B), shor diagonal (DA), long diagonal (DB)
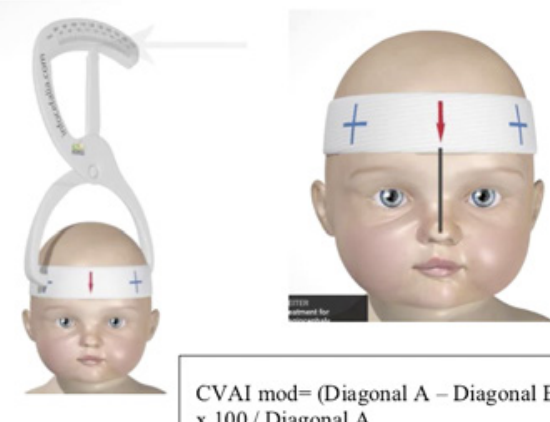

CVAI mod $=($ Diagonal $\mathrm{A}-$ Diagonal B $)$ x 100 / Diagonal A suring points of the skull.

General exclusion criteria were:

- Infants with prevalent febrile illnesses, infections, and vaccination reactions

- Previous osteopathic treatment

Specific exclusion criteria were:

- Symptomatic asymmetry (e.g., developmental dysplasia of the hip, perinatal fracture of the clavicle, congenital muscular torticollis)

- Synostotic plagiocephaly (due to craniosynostosis), concomitant helmet therapy

- Organic FD (e.g., due to neurological disorders, metabolic and gastrointestinal disorders)

- Crying infants older than 6 months, as well as crying due to diagnosed organic pain/a gastrointestinal problem

- Organic SD, and infants with FD aged less than 3 months

\section{Recruitment}

All 1,135 osteopaths from the official list of therapists by the largest German osteopathic professional association (Verband der Osteopathen Deutschland, VOD), who are indicated to specialize in the treatment of infants, were contacted in mid-December 2017, as well as 26 osteopaths from other associations. All participating osteopathic practitioners had an osteopathic training of at least $1,350 \mathrm{~h}$ (WHO standard), which reflects the standard of osteopathic training in Germany and were "Heilpraktiker," the only medical profession in Germany licensed to treat patients without supervision/delegation of a doctor, with special emphasis on complementary and alternative medicine. The prerequisite for consideration was a particular postgraduate training in the treatment of children fulfilling defined quality criteria.

Osteopaths consenting to take part were provided with complete information including information on the design of and flow through the study, a checklist of inclusion and exclusion criteria, forms to fill in concerning descriptive data and side effects, information leaflets and consent and other forms to pass on to the parents of infants to be included (see details below).

The inclusion of infants who met the inclusion criteria by participating practitioners had to be consecutive with those denying participation to be documented, too. It was mandatory for all participating practices to consecutively include all infants in the study who presented with one or more of the 5 health disturbances and who met the inclusion/exclusion criteria, once the first baby had been enrolled (documentation required, and checked upon return of forms and data sheets).

Outcome Measures

Based on literature research on the most commonly used clinical evaluation methods for the 5 health disturbances, specific symptom-oriented outcome assessment forms were designed for IA, EC, FD, and SD. Each form contained questions on the presence/absence of typical symptoms, and numerical rating scales (NRS: from $0=$ absent to $10=$ worst) [31] for quantification.

For IA, parents were asked for the preferred side for eye contact and a quantitative rating of the phenomenon on an NRS, the preferred side of rotation and a quantitative rating on an NRS, and whether they had the impression that their baby's typical lying position is asymmetric as much as head, chest and/or pelvis are concerned (yes/no and a quantitative rating on an NRS).

For EC, parents were asked to rate their own perceived level of stress due to their baby's crying (NRS: $0=$ no distress, $10=$ maximal distress), the effort necessary to calm their baby during a crying bout (NRS: $0=$ easily, $10=$ not possible), and how excessive the rate of the typical crying bout was (NRS: $0=$ normal, $10=$ most excessive).

For FD, parents were asked to indicate the presence/absence of six core problems during feeding, i.e., refusing to open the mouth, crying when offered food, exhaustion/sleep during feeding, sucking problems with breastfeeding, excessive feeding time, and to name other problems that they observed and a quantitative rating on an NRS $(0=$ not at all, $10=$ most pronounced $)$.

For SD, parents were asked to tick any of three major potential changes in sleeping habits, if considered crucial, namely difficulty falling asleep, staying asleep at night, and falling asleep again after awaking and a quantitative rating on an NRS $(0=$ not at all, $10=$ most difficult).

In DP, the Cranial Asymmetry Screening Measurement [32] was used to measure cranial asymmetry. The corresponding measuring points and measuring distances are shown in Figure 1. Based on these parameters, the cranial vault asymmetry index (CVAI), which reflects the extent of the lateral DP (pathological if CVAI $>3.5 \%$ [32]), was calculated.

\section{Intervention}

In keeping with the principles of osteopathy [1, 33, 34], there was no predefined, standardized treatment protocol; each osteopath was free to decide which techniques to use and how to document details according to the standard procedures of each practice. The number of osteopathic sessions and the time interval was not specified but had to be documented. The maximum observation/ study duration was limited to 90 days.

\section{Setting}

At the beginning of the first treatment session, inclusion and exclusion criteria were evaluated. The parents received comprehensive information about the study and were asked to sign a consent form. This was followed by the documentation of the initial 


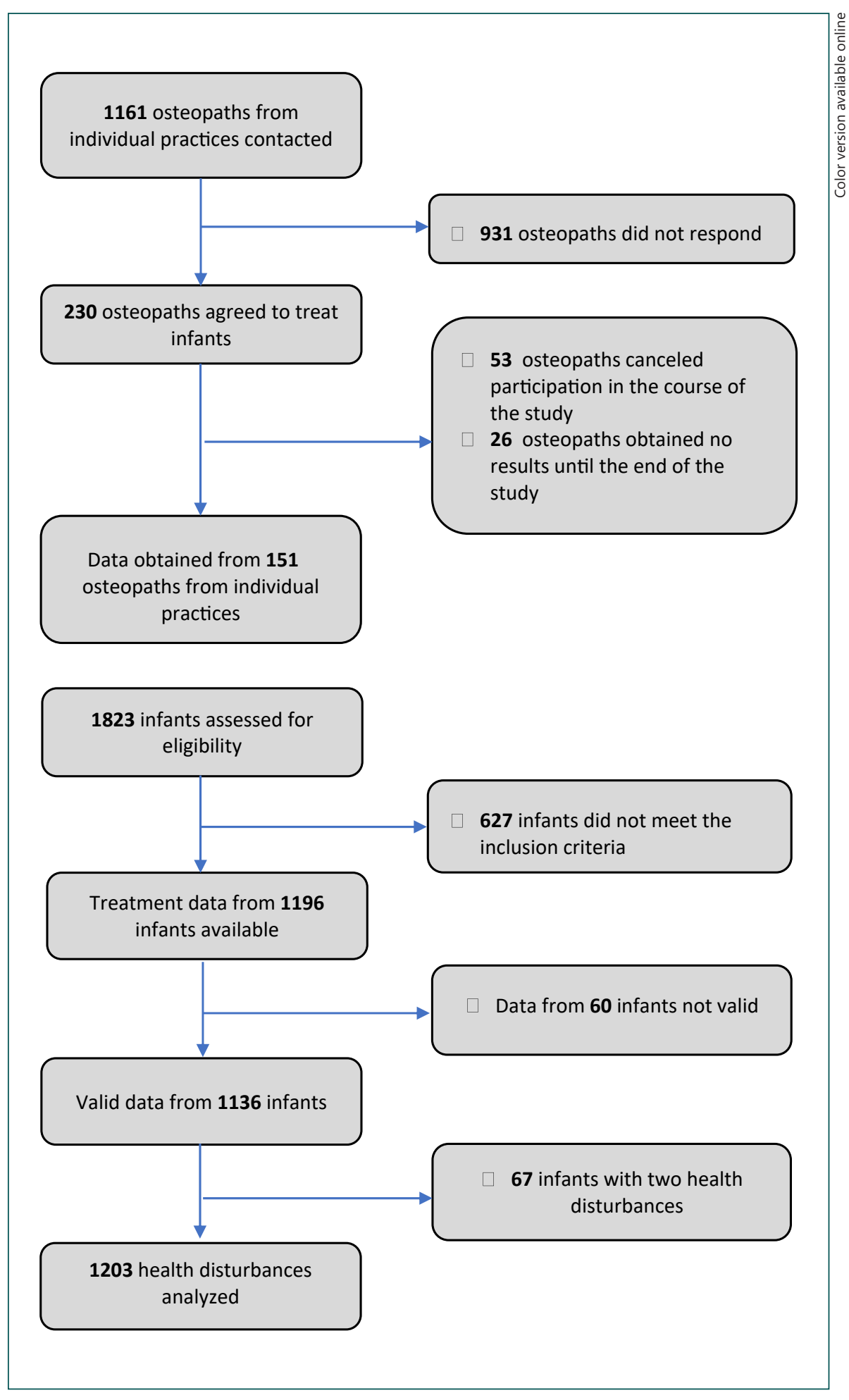

Fig. 2. Flow of subjects through the trial.

findings (baseline) and completion of the appropriate outcome assessment form by the parents. In the case of DP, the osteopath measured the child's skull and documented the results. For documentation purposes, standardized examination forms were used to monitor anamnestic data and osteopathic dysfunctions. After the last osteopathic treatment, the parents received the same outcome assessment form as at the beginning, which they were asked to fill in directly and leave in a closed envelope. In the case of DP, the osteopath measured the child's skull a second time.

\section{Data Management}

The participating osteopaths were asked to send all forms to the managing research organization (AFO) after the completion of their intervention phase. The forms were then checked for completeness, and the osteopaths were approached by telephone or mail, if additional information was required. Data were digitized using a popular spreadsheet program (MS Excel 2016), checked for typing errors, and subjected to plausibility control routines. 
Table 1. Baseline characteristics

\section{Gender $(n=1,136)$}

Age (mean $\pm \mathrm{SD})$, days

Mode of delivery

Recommendation for osteopathic treatment Nutrition

Osteopathic sessions (mean $\pm \mathrm{SD}$ ), $n$

Number of health disorders

( 1 disorder/2 disorders $n=1,069 / 67$ )
Female: 540 (48\%); male: 585 (51\%); not reported: 11 $72.5 \pm 59.3$

Vaginal: 70\%; assisted vaginal delivery: $9 \%$; cesarean: $30 \%$ Pediatrician: $18 \%$; midwife: $41 \%$; friends: $24 \%$; other: $16 \%$ Breast feeding: $59 \%$; bottle feeding: $25 \%$; both: $16 \%$ $3,212(2.8 \pm 1.1)$

Asymmetry: 582; plagiocephaly: 169 ; feeding disorders: 181; excessively crying infants: 219 ; sleeping disorders: 52

$\mathrm{SD}$, standard deviation.

Table 2. Baseline characteristics according to health disturbances

\begin{tabular}{|c|c|c|c|c|c|}
\hline Health disturbance characteristic & $\begin{array}{l}\text { Idiopathic } \\
\text { asymmetry }\end{array}$ & $\begin{array}{l}\text { Plagio- } \\
\text { cephaly }\end{array}$ & $\begin{array}{l}\text { Feeding } \\
\text { disorders }\end{array}$ & $\begin{array}{l}\text { Excessive } \\
\text { crying }\end{array}$ & $\begin{array}{l}\text { Sleeping } \\
\text { disorders }\end{array}$ \\
\hline Number $(n=1,203)$ & $582(48 \%)$ & $169(14 \%)$ & $181(15 \%)$ & $219(18 \%)$ & $52(4 \%)$ \\
\hline \multicolumn{6}{|l|}{ Gender, $n$} \\
\hline Female & 296 & 70 & 90 & 111 & 24 \\
\hline Male & 278 & 95 & 89 & 108 & 27 \\
\hline Not reported & 8 & 4 & 2 & 0 & 1 \\
\hline Age $($ mean $\pm S D)$, days & $68.3 \pm 48.2$ & $94.7 \pm 55.9$ & $49.8 \pm 46.2$ & $54.0 \pm 34.6$ & $212.3 \pm 9.6$ \\
\hline Number of osteopathic treatments (mean \pm SD) & $2.8 \pm 1.3$ & $3.2 \pm 1.0$ & $2.7 \pm 1.1$ & $2.8 \pm 1.0$ & $2.5 \pm 0.9$ \\
\hline
\end{tabular}

$\mathrm{SD}$, standard deviation.

Statistical Analysis

Due to the design of the study, i.e., hypothesis generating rather than hypothesis testing, all statistics are descriptive in nature. This is true, too, for the exploratory data analysis performed. Summary statistics were calculated for all recorded data and were presented as frequencies and percentages. Means and standard deviations were presented for continuous variables. Point estimates of changes were calculated and complemented by $95 \%$ confidence intervals.

\section{Ethics}

The study was conducted in accordance with the principles of Good Clinical Practice and the Declaration of Helsinki. The study protocol was approved by the institutional review board of the German Academy of Osteopathy.

\section{Results}

By the end of the recruitment period on February 1, 2018, 230 out of the 1,161 osteopaths contacted (19.8\%) had agreed to treat a total of approximately 1,710 infants; 118 of those indicated on a short questionnaire that they were prepared to treat 5 infants, 72 to treat 10 infants, and 40 to treat more than 10 infants. At the end of the study period, 151 osteopaths had participated and treated 1,196 infants in the study (Fig. 2). Data sheets of 60 infants could not be included in the analysis (for details see Discussion).

Tables 1 and 2 show the baseline characteristics of the infants included in the study. Five hundred and eightytwo infants (48\%) were diagnosed with IA, $219(18 \%)$ with EC, 181 (15\%) with FD, 169 (14\%) with DP, and 52 (4\%) with SD. More than one diagnosis was made in 67 infants. A total of 3,212 treatment sessions were documented for the 1,136 infants included in the analysis, corresponding to an average treatment frequency of 2.8 treatments per infant $(n=83$ infants received 1 treatment, $n=790$ received 2 treatments, $n=1,182$ received 3 treatments, $n=680$ received 4 treatments, $n=435$ received 5 treatments, and $n=42$ received 6 treatments).

\section{Results of the Individual Health Disturbances}

The results for IA and DP are shown in detail in Table 3. Of a total of 582 IA-diagnosed infants, 427 (84\%) had an asymmetric position of the head, thorax, or pelvis. Overall, symptoms improved on average from about baseline values of somewhat over 6 to somewhat over 1 on the NRS for all three areas combined.

For DP, 37 out of 169 cases (22\%) had a CVAI that was in the normal range $(<3.5 \%)$ at baseline. These cases were not included in the evaluation. Furthermore, results re- 
Table 3. Change of symptoms for idiopathic asymmetry and plagiocephaly

\begin{tabular}{|c|c|c|c|c|c|c|}
\hline & \multicolumn{2}{|c|}{ Baseline symptom(s) } & \multirow{2}{*}{$\begin{array}{l}\text { Baseline } \\
\text { mean } \pm \text { SD }\end{array}$} & \multirow{2}{*}{$\begin{array}{l}\text { End of treatment } \\
\text { mean } \pm S D\end{array}$} & \multirow{2}{*}{$\begin{array}{l}\text { Difference of } \\
\text { longitudinal } \\
\text { changes }(95 \% \mathrm{CI})\end{array}$} & \multirow{2}{*}{$\begin{array}{l}\text { Improvement, } \\
\%\end{array}$} \\
\hline & right & left & & & & \\
\hline \multicolumn{7}{|l|}{ Idiopathic asymmetry $(n=582)$} \\
\hline $\begin{array}{l}\text { Eye contact to the preferred } \\
\text { side intensity (NRS } 0-10)\end{array}$ & 346 & 230 & $6.5 \pm 1.8$ & $1.3 \pm 1.5$ & $5.2(4.8-5.6)$ & 79 \\
\hline $\begin{array}{l}\text { Rotation to the preferred } \\
\text { side intensity (NRS } 0-10 \text { ) }\end{array}$ & 204 & 140 & $6.2 \pm 1.9$ & $1.3 \pm 1.6$ & & 78 \\
\hline Asymmetric position & 204 & 140 & $0.2 \pm 1.9$ & $1.0 \pm 1.0$ & $4.8(4.0-5.2)$ & 18 \\
\hline intensity (NRS 0-10) & $427^{1}$ & & $6.1 \pm 2.0$ & $1.1 \pm 1.4$ & $5.0(4.8-5.2)$ & 82 \\
\hline \multicolumn{7}{|l|}{ Plagiocephaly $(n=119)$} \\
\hline CVAI, \% & n.a. & & $8.0 \pm 3.1$ & $4.0 \pm 2.7$ & $4.0(3.5-4.5)$ & 51 \\
\hline
\end{tabular}

CI, confidence interval; NRS, numerical rating scale; SD, standard deviation; CVAI, cranial vault asymmetry index; n.a., not available. ${ }^{1}$ For both right and left.

Table 4. Change of symptoms for feeding disorders, excessively crying infants and sleep disorders

\begin{tabular}{|c|c|c|c|c|}
\hline & $\begin{array}{l}\text { Baseline } \\
\text { mean } \pm \text { SD }\end{array}$ & $\begin{array}{l}\text { End of treatment } \\
\text { mean } \pm S D\end{array}$ & $\begin{array}{l}\text { Difference of } \\
\text { longitudinal } \\
\text { changes }(95 \% \mathrm{CI})\end{array}$ & $\begin{array}{l}\text { Improvement, } \\
\%\end{array}$ \\
\hline \multicolumn{5}{|l|}{ Feeding disorders $(n=181)$} \\
\hline \multicolumn{5}{|l|}{ Questions about the number of problems that have } \\
\hline Intensity (NRS 0-10) & $7.3 \pm 1.5$ & $1.7 \pm 1.8$ & $5.6(5.3-5.9)$ & 77 \\
\hline \multicolumn{5}{|l|}{ Excessively crying infants $(n=219)$} \\
\hline Perceived stress level (NRS 0-10) & $7.0 \pm 1.9$ & $2.3 \pm 1.6$ & $4.7(4.4-5.0)$ & 67 \\
\hline Difficulty to calm (NRS 0-10) & $6.8 \pm 2.0$ & $2.0 \pm 1.6$ & $4.8(4.5-5.1)$ & 70 \\
\hline Excessive crying (NRS 0-10) & $7.7 \pm 1.7$ & $2.3 \pm 1.8$ & $5.4(5.1-5.7)$ & 70 \\
\hline \multicolumn{5}{|l|}{ Sleeping disorders $(n=52)$} \\
\hline \multicolumn{5}{|l|}{ Questions about the number of problems that have } \\
\hline Intensity (NRS $0-10$ ) & $7.7 \pm 1.4$ & $3.4 \pm 2.0$ & $4.4(3.8-5.0)$ & 56 \\
\hline
\end{tabular}

SD, standard deviation; CI, confidence interval; NRS, numerical rating scale.

ported for 13 infants (8\%) were considered "outliers," probably due to problems in the application of the craniometer. These cases were not included in the evaluation. For the 119 cases evaluated, the CVAI improved from $8.0 \%$ at the beginning to $4.0 \%$ at the end, which corresponds to an improvement of $51 \%$ and a broad approximation to the normal range.

The problem of EC was seen in 219 infants and was considered the second most frequent reason for consultation in a participating osteopathic practice. Infants with FD ( $n=181)$ formed the youngest group with about 50 days of age, and SD were the least frequent and found in 52 infants (4\%) of this study. In all 3 of these health disturbances, a significant improvement of the initial values of about $75 \%$ on the NRS to final values of about
$25 \%$ was observed. The individual results are shown in Table 4.

Of the 1,136 infants treated, 90 showed signs of intercurrent illness (i.e., coughs and colds) between the 1st and 2nd treatment, 55 between the 2nd and 3rd treatment, 22 between the $3 \mathrm{rd}$ and 4 th treatment, and 5 between the 4 th and 5 th treatment. The required intake of medication during the treatment period was very low; only 21 infants needed medication.

Adverse reactions with a time-related reference to osteopathic sessions occurred in only 75 of the 1,136 infants and 3,212 treatments, respectively. Sixty-three infants showed adverse reactions after the first treatment, 11 infants after the second treatment, and 1 infant after the third treatment. Reactions represented typical daytime 
variations, e.g., restlessness, fatigue, restless sleep, mild diarrhea, or flatulence. Clinically relevant, serious, or nontransient side effects did not occur during the study.

\section{Discussion}

\section{Study Design}

This prospective, multicenter, observational study was conducted between January 2017 and December 2018, with the observation period starting in February 2018. The objective of this study was not to quantify the efficacy of an osteopathic intervention, as it would be in a randomized controlled trial, but rather to observe and document changes in the prevalence/extent of symptoms of qualifying disorders/symptoms/health concerns during the course of treatment as perceived by parents and treating osteopaths. The method of choice for this scenario was that of an observational study, suitable to realistically depict the real-life situation in daily medical practice [35].

In Germany, as in most other countries in the Western world, it can be assumed that the spontaneously perceived health-related abnormalities (health disorders/ concerns) of infants are first diagnosed and, if necessary, treated within the framework of the respective care system. It can further be assumed that in countries such as Germany, where osteopathy is not an integral part of the primary care system or reimbursable subject-specific care, the presentation of a child in an osteopathic practice is usually a subsidiary step initiated by the parents if usual care did not have the expected success. Against this background of a "client-driven initiative," it seemed sensible to take the parents' point of view and invite them to assess the (perceived) outcome, rather than that of the health care system which had probably not been able to produce a subjectively satisfactory outcome before in order to produce (pragmatic) meaningful results.

Terminology on osteopathy is not consistent. "Osteopathic manual treatment" (OMT) is by far the most commonly used term found in Medline to describe the typical therapeutic scenario taking place in an osteopathic practice, yet coined in the USA and implying features typical for practices in the USA (such as integral part of the health care system, primarily biomedical diagnostic procedures, typically applying a predefined set of osteopathic techniques). Since the typical environment and features in a European (German) osteopathic practice are greatly different from those in the USA in various aspects, European osteopaths prefer not to use the term "OMT" but, for instance, "osteopathic treatment" or "osteopathic intervention" in order to indicate the potential presence of major differences as compared with the US setting.

\section{Methods}

The approach of including a large number of study subjects and involving the largest possible number of study centers requires a smart and pragmatic approach well adapted to common practice procedures and hardly interfering with the normal clinical process. In addition, this approach may minimize a possible study bias potentially caused by special attention (the so-called Hawthorne effect $[36,37])$. Since this is not a confirmatory study, a sample size estimation was neither necessary nor useful. The desired scope had to be determined on the premise that the most reliable and meaningful findings would be achieved.

The exclusion criterion of fewer than 6 months of age among excessively crying infants was based on the literature, as beyond this age a reduction in the duration of crying is commonly seen and reported [25]. For SD, only infants older than 3 months were admitted, as irregular sleeping patterns are particularly common in the first postpartum period.

\section{Outcome Measures}

The different clinical features/symptoms of the 5 included health disturbances made the use of different outcome measures necessary. The outcome measures used in the study were largely based on the results of extensive literature analyses, which resulted in the finding that simple, reliable, and valid standards appeared to be lacking for 4 of the 5 health disturbances.

\section{Idiopathic Asymmetry}

In order to be able to accurately document and evaluate the changes that occur under the applied therapy, a highly objective clinical method of measurement is required. Unfortunately, there are hardly any common, easy to perform, and well-recognized methods of assessment in the literature [6].

\section{Feeding Disorders}

Various studies and reviews $[38,39]$ examined instruments for the evaluation of feeding in infants, for example the so-called Feeding Scale. They all concluded that all existing instruments are to be considered with caution and are complicated to use from the parents' point of view.

\section{Excessive Crying}

Various methods have been developed for the documentation of crying bouts, e.g., tape recordings, recording of the duration of crying with the validated 24-h crying diary, or questionnaires such as the Crying Pattern Questionnaire or the Infant Crying Questionnaire [40, 41]. All methods, however, are very time consuming for parents. 
Sleeping Disorders

Sleeping behavior can be documented with a sleep protocol that records the duration of sleep as well as sleeping/waking times every $15 \mathrm{~min}$ over a time period of $24 \mathrm{~h}$. However, it was deemed unrealistic to expect that parents systematically adhere to such a protocol over several weeks.

When selecting the outcome assessments for this study, the primary focus of these 4 health disturbances was therefore on the observations, along with their subjective evaluation by the parents, and on easy-to-perform documentation. The questions had to be answered as conveniently as possible by the parents, reflecting their personal assessment of the treatment period as reliably and consistently as possible. For the participating osteopaths, a latent scientific inhibition threshold had to be minimized and the daily routine of treatments given was to be disturbed as little as possible. Against this background, an NRS, a common generic measurement tool [31], evolved as the best possible instrument.

The assessment of the fifth health disturbance, plagiocephaly, was performed by means of a craniometer. It is a fast and simple 2-dimensional measuring technique that the osteopath can perform with a caliper. This tool is useful for diagnosing DP and for monitoring the course of therapy [42], although some of the participating osteopaths objected to the precision of that measurement for a single course. However, in assessing possible changes, this instrument appeared to be sufficiently suitable for the present study.

\section{Selection Bias}

The start of the study was individually determined in each practice, with all infants who presented with one of the 5 health disturbances being documented. Included in the study were only those infants who met all inclusion criteria and for whom, in addition, the consent form was signed by the parents. This was to ensure that a consecutive study intake was carried out and that potential selection bias was avoided as much as possible. A total of 1,823 consecutive infants were assessed, of whom 1,196 (65\%) met all inclusion criteria. An assessment of the study documentation of each of the 151 participating osteopaths indicated that 101 osteopaths probably complied sufficiently with the predefined recruitment framework, whereas in 50 osteopaths, the respective evidence did not appear to be sufficiently likely according to their study documentations. A sensitivity analysis in infants included for IA, which compared the results of all 151 participating osteopaths to those of the 101 osteopaths with compliant study documentation with respect to the recruitment framework, did not, however, reveal any significant differences.

\section{Intervention}

Osteopathic practitioners use their understanding of the relationship between structure and function to optimize the body's self-regulation and self-healing capabilities. This approach to patient care and treatment is based on the concept that a human being is a dynamic functional unit, in which all parts are interrelated and which possesses its own self-regulatory and self-healing mechanisms. Two essential components of osteopathic health care are the structural evaluation of the patient for diagnosis and an array of manipulative techniques for treatment [1]. The aim of the structural examination is to locate somatic dysfunctions that may contribute to the clinical presentation of the patient. Diagnostic criteria for somatic dysfunctions are focused on tissue texture abnormalities, asymmetry of bony landmarks and restriction of motion [33].

Osteopathic practitioners use a wide variety of therapeutic manual techniques to improve physiological function and restore homeostasis impaired by somatic dysfunction [1]. They assess and treat the "whole person" as a self-regulatory system, rather than just focusing on specific symptoms or illnesses [34]. According to the principles of osteopathy, the location of dysfunction will not be restricted to the area of symptoms; dysfunctions can arise and be diagnosed throughout the body. At every osteopathic treatment session, only those structures for which actual osteopathic findings are present will be treated.

According to the Glossary of Osteopathic Terminology [33], OMT typically involves a range of manual techniques. When treating infants, however, balanced ligamentous tension and cranial treatments (osteopathy in the cranial field/cranial osteopathy) are the treatment approaches of choice.

\section{Results}

The willingness of osteopaths to participate in the study was unexpectedly high. The originally planned number of 500 infants to be treated was exceeded 3 times. This strongly suggests that the topic of the study interested many osteopaths, who were encouraged to contribute to the evidence by taking part in this study. Unfortunately, during the study, 53 osteopaths dropped out, primarily due to illness, family problems, or relocation. Additionally, during the study period, the number of infants presented suddenly declined dramatically for no particular reason, an effect that is not unlikely to be observed in clinical trials. Sixty data records were not evaluable, in most cases because the parents did not appear for the second or last treatment. The resulting dropout rate of $5 \%$ compares well with other clinical studies.

The vast majority of osteopathic practices are singlehanded, and some centers are run by 2 or more osteo- 
paths, but there are no larger osteopathic units comparable to clinics within the health care system. The participating osteopaths had an average of 12 years of work experience (minimum 3 years, maximum 23 years). The gender distribution of infants was balanced (female $48 \%$, male $51 \%$ ). None of the health disturbances were noticeably gender related. According to the data of the German Federal Statistical Office, in 2015 the birth rate due to cesarean deliveries was $31 \%$, corresponding to the distribution of cesarean sections in our study (30\% cesarean section, $70 \%$ vaginal delivery). The average age of the infants was 72 days (minimum 5 days, maximum 362 days). The young age of the infants presented suggests that the health disturbances included in this study cause much concern for parents at a very early age, and that most parents decide to seek help from an osteopath early on. On average, participating osteopaths treated 7.5 infants each. The number of treatment sessions was not fixed, as it depended on the different circumstances of each infant and was individualized according to the infant's needs, ranging from 1-2 treatments to 5 or more. Also, the time interval between consecutive treatment sessions varied and depended largely on the health of the infant (e.g., a sudden cold). As the changes in symptoms were assessed before the last osteopathic treatment, the potential effects of the last osteopathic treatment were not covered by the analysis, which would result in an underestimation of treatment effects rather than an overestimation.

IA was the most common health disorder in this study. The observations that boys are more frequently affected than girls and that the more common preferential direction of the asymmetry is to the right side are in line with findings published elsewhere [43]. Likewise, with DP, boys are more often affected than girls. On average, 3.2 treatments were needed to address this health disturbance. We are unable to comment on the question of which side presents more often with cranial flattening, as the respective information was not collected. Correlations as to whether the IA and DP frequently occur in cesarean sections or assisted births (e.g., forceps, ventouse) were not observed.

In the case of FD, the clinical findings of the various problems encountered (6 questions to be addressed by the parents) improved to the same extent as the intensity of the symptoms, which corroborates the validity of the results. The parents' ratings on the NRS for FD, EC, and SD were both high (greater than 7 out of 10), which at least indicates how much these infant disorders affect parents. A detailed presentation of the documented osteopathic findings would have exceeded the scope of this publication.

The repeated accusations presented in the media that osteopathic medicine would harm the health of infants was not confirmed in this numerically largest study to date. There was not a single significant/serious adverse reaction of an infant to the osteopathic treatment given.

\section{Conclusions}

There seems to be remarkably high demand among parents for the osteopathic treatment of infants in Germany, which may indicate that the perceived unsatisfactory results of conventional care options for these problems are, at least, not uncommon.

A small series of osteopathic sessions performed by well-educated osteopaths with specific postgraduate education was associated with marked positive changes in the severity of the 5 major childhood disorders identified by parents (IA, DP, FD, EC, and SD) and, in most cases, resolved as a matter of concern.

The prospective observation of an application or therapy as performed in this study does not claim to establish causality between an intervention and timely associated effects in the narrower sense, nor to derive a quantitative estimation of the effect of osteopathic treatment on infants. It may, however, given the large number of participants (this happens to be, to our knowledge, as yet one of the largest observational studies on the topic in the world) and a successful effort to minimize selection bias (e.g., through documented recording), yield qualitatively useful results.

In essence, all 5 health concerns included in this study proved to be promising candidates for further clinical investigations, in particular for randomized controlled trials into the efficacy and effectiveness of osteopathic treatment for infants.

\section{Acknowledgment}

Thanks go to Dr. Anne Jaekel for translation of this manuscript into English.

\section{Statement of Ethics}

The study protocol was approved by the Institutional Review Board of the German Academy of Osteopathy (EK005, January 2018). Written informed consent was obtained from all parents before their infants were enrolled in the study. The study was conducted in accordance with the principles of Good Clinical Practice and the Declaration of Helsinki. The study protocol was approved by the institutional review board of the German Academy of Osteopathy.

The study was registered in the German Clinical Trials Register: DRKS00013137, registration date January 18, 2018 (https:// www.drks.de/drks_web/navigate.do? navigationId = trial.HTML\& TRIAL_ID = DRKS00013137). 


\section{Conflict of Interest Statement}

The authors have no conflicts of interest to declare. No benefits in any form have been or will be received from a commercial party related directly or indirectly to the subject of this paper.

\section{Funding Sources}

The study was sponsored by the German Osteopathic Associations "Verband der Osteopathen Deutschland" (VOD), "Register der traditionellen Osteopathen in Deutschland" (ROD), "Bundesverband Osteopathie" (BVO), and "Förderverein für osteopa- thische Forschung" (FOF). The funding bodies did not influence the design of the study, analysis, and interpretation of data and in writing the paper.

\section{Author Contributions}

F.S., K.L.R., E.M., and B.S. contributed to the design and planning of the research. E.M. and B.S. helped in the development of the outcome assessments, F.S. carried out the data collection and was involved in the analysis of results. F.S. and K.L.R. drafted the manuscript. All authors were involved in the critical revision of the paper.

\section{References}

1 World Health Organization (WHO). Benchmarks for Training in Osteopathy. 2010 [cited 2018 Nov 15]. Available from http://www. who.int/medicines/areas/traditional/BenchmarksforTraininginOsteopathy.pdf.

2 Osteopathie in Deutschland. 2018 [Cited 15.11.2018]. Available from: https://www.osteopathie.de/forsa_umfrage.

3 Posadzki P, Lee MS, Ernst E. Osteopathic manipulative treatment for pediatric conditions: a systematic review. Pediatrics. 2013 Jul; 132(1):140-52

4 Hayden C, Mullinger B. A preliminary assessment of the impact of cranial osteopathy for the relief of infantile colic. Complement Ther Clin Pract. 2006 May;12(2):83-90.

5 Heber A, Senger U. Osteopathic treatment of infantile colic [Thesis]. Gauting: German Academy of Osteopathy; 2003. Abstract available from: https://www.osteopathie-akademie.de.

6 Philippi H, Faldum A, Jung T, Bergmann H, Bauer K, Gross D, et al. Patterns of postural asymmetry in infants: a standardized videobased analysis. Eur J Pediatr. 2006 Mar; 165(3):158-64.

7 Philippi H, Faldum A, Schleupen A, Pabst B, Jung T, Bergmann $\mathrm{H}$, et al. Infantile postural asymmetry and osteopathic treatment: a randomized therapeutic trial. Dev Med Child Neurol. 2006 Jan;48(1):5-9.

8 Niggemeier $H$, Wilke $H$. Have osteopathic treatments a positive effect on the extent of the pathology of a congenital, muscular torticollis on infants? A randomized controlled trial [Thesis]. Gauting: German Academy of Osteopathy; 2005. Abstract available from: https://www.osteopathie-akademie.de.

9 Brockmeyer I, Exner-Panne K, Peschke K. Evaluation of the effectiveness of osteopathic and physiotherapeutic intervention on idiopathic infantile asymmetry. A randomized controlled trial [Thesis]. Gauting: German Academy of Osteopathy; 2009. Abstract available from: https://www.osteopathie-akademie.de.

10 Hasemann B, Kretschmer U, Lange T. Osteopathic treatment compared to physiotherapy in infants with idiopathic postural asymmetry. A randomized controlled trial [Thesis]. Gauting: German Academy of Osteopathy;
2016. Abstract available from: https://www. osteopathie-akademie.de.

11 Lund G, Carreiro JE. Characteristics of pediatric patients seen in medical school-based osteopathic manipulative medicine clinics. J Am Osteopath Assoc. 2010 Jul;110(7):376-80.

12 Mellitzer E. Which problems make parents approaching an osteopath for their children in their first year of life and which somatic dysfunctions can be found? [MSc thesis]. Krems: Danube University Krems; 2014. Available from: http://www.osteopathicresearch.com.

13 Schwerla F, Daake B, Möckel E, Resch KL. Most common infant health disorders in osteopathic practices in Germany. Survey (Lond). Submitted 2019.

14 van Vlimmeren LA, Helders PJ, van Adrichem LN, Engelbert RH. Diagnostic strategies for the evaluation of asymmetry in infancy-a review. Eur J Pediatr. 2004 Apr;163(4-5): 185-91.

15 Dobson D, Lucassen PL, Miller JJ, Vlieger AM, Prescott P, Lewith G. Manipulative therapies for infantile colic. Cochrane Database Syst Rev. 2012 Dec;12:CD004796.

16 Nuysink J, van Haastert IC, Takken T, Helders PJ. Symptomatic asymmetry in the first six months of life: differential diagnosis. Eur J Pediatr. 2008 Jun;167(6):613-9.

17 Looman WS, Flannery AB. Evidence-based care of the child with deformational plagiocephaly, Part I: assessment and diagnosis. J Pediatr Health Care. 2012 Jul-Aug;26(4):24250.

18 Flannery AB, Looman WS, Kemper K. Evidence-based care of the child with deformational plagiocephaly, part II: management. J Pediatr Health Care. 2012 Sep-Oct;26(5): 320-31.

19 Laughlin J, Luerssen TG, Dias MS; Committee on Practice and Ambulatory Medicine, Section on Neurological Surgery. Prevention and management of positional skull deformities in infants. Pediatrics. 2011 Dec;128(6): 1236-41.

20 Flannery AM, Tamber MS, Mazzola C, Klimo P Jr, Baird LC, Tyagi R, et al. Congress of Neurological Surgeons Systematic Review and Evidence-Based Guidelines for the Management of Patients With Positional Plagiocephaly: ex- ecutive Summary. Neurosurgery. 2016 Nov; 79(5):623-4.

21 Hutchison BL, Hutchison LA, Thompson JM Mitchell EA. Plagiocephaly and brachycephaly in the first two years of life: a prospective cohort study. Pediatrics. 2004 Oct;114(4): 970-80.

22 Bialocerkowski AE, Vladusic SL, Wei Ng C. Prevalence, risk factors, and natural history of positional plagiocephaly: a systematic review. Dev Med Child Neurol. 2008 Aug;50(8):577-86.

23 Martiniuk AL, Vujovich-Dunn C, Park M, Yu W, Lucas BR. Plagiocephaly and Developmental Delay: A Systematic Review. J Dev Behav Pediatr. 2017 Jan;38(1):67-78.

24 Wessel MA, Cobb JC, Jackson EB, Harris GS Jr, Detwiler AC. Paroxysmal fussing in infancy, sometimes called colic. Pediatrics. 1954 Nov; 14(5):421-35.

25 Akhnikh S, Engelberts AC, van Sleuwen BE, L'Hoir MP, Benninga MA. The excessively crying infant: etiology and treatment. Pediatr Ann. 2014 Apr;43(4):e69-75.

26 Helseth S, Begnum S. A comprehensive definition of infant colic: parents' and nurses' perspectives. J Clin Nurs. 2002 Sep;11(5):672-80.

27 Rybak A. Organic and nonorganic feeding disorders. Ann Nutr Metab. 2015;66 suppl 5: 16-22.

28 Papoušek M, Scholtes $\mathrm{K}$, Rothenburg S, von Hofacker N, Cierpka M. Ein- und Durchschlafstörungen in den ersten beiden Lebensjahren. Monatsschr Kinderheilkd. 2009;157(5):483-92.

29 Papousek M. [Regulatory disorders in early childhood. Family physician counseling for crying, sleeping and feeding disorders]. MMW Fortschr Med. 2005 Mar;147(12):324. German.

30 AWMF online. S2k-Leitlinie 028/041 - Psychische Störungen im Säuglings-, Kleinkindund Vorschulter. 2015 [cited 2018 Nov 15]. Available from: https://www.awmf.org/uploads/tx_szleitlinien/028-0411_S2k_Psychische_Stoerungen_Saeugling_Kleinkind_ Vorschulalter_2017-10.

31 Castarlenas E, Jensen MP, von Baeyer CL, Miró J. Psychometric Properties of the Numerical Rating Scale to Assess Self-Reported Pain Intensity in Children and Adolescents: A Systematic Review. Clin J Pain. 2017 Apr; 33(4):376-83. 
32 Dörhage KW, Beck-Broichsitter BE, von Grabe V, Sonntag A, Becker ST, Wiltfang J. Therapy effects of head orthoses in positional plagiocephaly. J Craniomaxillofac Surg. 2016 Oct;44(10):1508-14.

33 Ward RC, editor. Foundations of osteopathic medicine. 2nd ed. Philadelphia: Lippincott Williams \& Wilkins; 2003.

34 Osteopathic International Alliance (OIA). Global Osteopathic Reports. 2014 [cited 2018 Nov 15]. Available from https://oialliance. org/resources/oia-status-report/.

35 Papanikolaou PN, Christidi GD, Ioannidis JP. Comparison of evidence on harms of medical interventions in randomized and nonrandomized studies. CMAJ. 2006 Feb;174(5): 635-41.
36 Parsons H. What happened at Hawthorne? Science 1974; 183:922-32.

37 Sedgwick P, Greenwood N. Understanding the Hawthorne effect. BMJ. 2015 Sep; 351:h4672.

38 Pados BF, Park J, Estrem H, Awotwi A. Assessment Tools for Evaluation of Oral Feeding in Infants Younger Than 6 Months. Adv Neonatal Care. 2016 Apr;16(2):143-50.

39 Chatoor I, Getson P, Menvielle E, Brasseaux C, O'Donnell R, Rivera Y, et al. A feeding scale for research and clinical practice to assess mother-infanti interactions in the first three years of life. Infant Ment Health J. 1997;18(1): 76-91.
40 Lucassen PL, Assendelft WJ, van Eijk JT, Gubbels JW, Douwes AC, van Geldrop WJ. Systematic review of the occurrence of infantile colic in the community. Arch Dis Child. 2001 May;84(5):398-403.

41 Wolke D, Meyer R, Gray P. Validity of the crying pattern questionnaire in a sample of excessively crying babies. J Reprod Infant Psychol. 1994;12(2):105-14.

42 Öhman A. A craniometer with a headband can be a reliable tool to measure plagiocephaly and brachycephaly in clinical practice. Health. 2016;8(8):1258-65.

43 Boere-Boonekamp MM, van der Linden-Kuiper LT LT. Positional preference: prevalence in infants and follow-up after two years. Pediatrics. $2001 \mathrm{Feb} ; 107(2): 339-43$. 